\title{
miR-365b-3p inhibits the cell proliferation and migration of human coronary artery smooth muscle cells by directly targeting ADAMTS1 in coronary atherosclerosis
}

\author{
YUNFEI QU $^{1}$ and NING ZHANG ${ }^{2}$ \\ Departments of ${ }^{1}$ Cardiac Vascular Surgery and ${ }^{2}$ General Medicine, \\ Chongqing Three Gorges Central Hospital, Chongqing 404000, P.R. China
}

Received April 25, 2018; Accepted August 2, 2018

DOI: $10.3892 /$ etm.2018.6720

\begin{abstract}
Abnormal proliferation and migration of vascular smooth muscle cells serves a crucial role in the development of atherosclerosis. Previous studies have suggested that some microRNAs (miRs) are involved in this process; however, the associated underlying molecular mechanism is unclear. In present study, human coronary artery smooth muscle cells (HCASMCs) were used to explore the function of miR-365b-3p in the coronary atherosclerosis. It was indicated that platelet-derived growth factor-BB (PDGF-BB) treatment inhibited miR-365b-3p expression and upregulated the expression of a disintegrin and metalloproteinase with thrombospondin motifs 1 (ADAMTS1) in HCASMCs. Subsequently, miR-365b-3p mimic was transfected in HCASMCs to explore the function of this miR. The results of reverse transcription-quantitative polymerase chain reaction and western blot analysis indicated that overexpression of miR-365b-3p significantly downregulated ADAMTS1 expression.Functionalassay results revealed that overexpression of miR-365b-3p significantly attenuated PDGF-BB-induced proliferation and migration of HCASMCs. Furthermore, the dual-luciferase reporter assay results confirmed that ADAMTS1 is a direct target gene of miR-365b-3p. This discovery proposed a novel channel of communication between ADAMTS1 and HCASMCs, and suggests a potential therapeutic approach for coronary atherosclerosis.
\end{abstract}

Correspondence to: Dr Ning Zhang, Department of General Medicine, Chongqing Three Gorges Central Hospital, 165 Xincheng Road, Chongqing 404000, P.R. China

E-mail: zhangning99@aliyun.com

Key words: microRNA-365b-3p, human coronary artery smooth muscle cells, A disintegrin and metalloproteinase with thrombospondin motifs 1 , coronary atherosclerosis

\section{Introduction}

Coronary atherosclerosis is a chronic disease characterized by ongoing progression in response to systemic risk factors and local pro-atherogenic stimuli (1). Atherosclerosis is the primary cause of coronary heart disease, cerebral infarction and peripheral vascular disease (2). Notably, atherosclerotic cardiovascular disease remains the leading cause of human fatality worldwide (3). Vascular smooth muscle cells (VSMCs) have a critical role in atherosclerotic plaque formation. Abnormal proliferation and migration of VSMCs within the intima contributes to initial atherosclerotic plaque formation (4). Notably, VSMCs can form fibrous caps to stabilize vulnerable plaques at advanced stages (5). Furthermore, a previous study has indicated the regulation of atherosclerosis at tissue and molecular levels is associated with VSMCs (6). Several reports have demonstrated that platelet-derived growth factor-BB (PDGF-BB) can stimulate VSMC proliferation and migration during vascular injury $(7,8)$. Therefore, exploring the underlying molecular mechanism involved in the modulation of PDGF-BB-dependent VSMC proliferation and migration, including PDGF-BB-dependent human coronary artery smooth muscle cell (HCASMC) proliferation and migration, may be of great scientific interest.

MicroRNAs (miRs) are a type of endogenous non-coding RNA that are 22 nucleotides in length (9). By binding directly to the 3'-untranslated region (UTR) of target mRNAs, miRs contribute to mRNA degradation or transcriptional inhibition, thus regulating gene expression (10). Accumulating evidence has indicated that miRs serve an important role in VSMC proliferation, migration and remodeling. For example, miR-26a is significantly increased following PDGF-BB treatment in VSMCs, and is involved in VSMC phenotypic transition by targeting Smad1 (11). Notably, miR-24 effectively regulated vascular remodeling and reduced the level of inflammatory factors in a diabetic rat model (12). A previous study indicated that overexpression of miR-612 inhibited VSMC proliferation and migration through inducing cell cycle arrest at G1 stage, and RAC- $\beta$ serine/threonine-protein kinase (AKT2) was identified as a direct target of miR-612 (13). Furthermore, miR-448 has been identified to be highly expressed in VSMCs from coronary atherosclerotic plaques compared with normal 
VSMCs (14). Notably, miR-448 promoted the proliferation and migration of VSMCs by targeting myocyte enhancer factor $2 \mathrm{C}$ (14). There are fewer reports concerning the role of miR-365b-3p. A recent study indicated that overexpression of miR-365b-3p upregulated p21 and p27 and induced cell cycle arrest in $\mathrm{G}_{1}$ phase and cell apoptosis (15). However, the role of miR-365b-3p in the HCASMCs is poorly understood.

A disintegrin and metalloproteinase with thrombospondin motifs 1 (ADAMTS1) is the member of the ADAMTS family, a family of extracellular proteases (16). ADAMTS1, which is regulated by peroxisome proliferator-activated receptor, promotes the proliferation and migration of VSMCs, inducing atherosclerosis and vascular thrombosis (17). A recent study suggested that miR-362-3p directly binds to ADAMTS1 and inhibits the proliferation and migration of VSMCs (18). However, there is no research to clarify the association between ADAMTS1 and miR-365b-3p. In present study, the effect of PDGF-BB treatment was explored on miR-365b-3p and ADAMTS1 expression in HCASMCs. Functional assays were performed to assess cell the proliferation and migration of HCASMCs. TargetScan Human 3.1 (www.targetscan.org) and the dual-luciferase reporter assay were used to identify whether ADAMTS1 is a potential target of miR-365b-3p.

\section{Materials and methods}

Cell culture and treatment. HCASMCs (cat. no. C0175C; Thermo Fisher Scientific, Inc., Waltham, MA, USA) were cultured in Dulbecco's modified Eagle's medium (DMEM; Thermo Fisher Scientific, Inc.) supplemented with $10 \%$ fetal bovine serum (Thermo Fisher Scientific, Inc.) at $37^{\circ} \mathrm{C}$ in a humidified atmosphere containing $5 \% \mathrm{CO}_{2}$. For the PDGF-BB group, HCASMCs were treated with $30 \mathrm{ng} / \mathrm{ml}$ PDGF-BB (Thermo Fisher Scientific, Inc.) for $6 \mathrm{~h}$ at $37^{\circ} \mathrm{C}$.

Transfection and groups. miR-365b-3p mimic and negative control (NC) mimic were obtained from GenePharma (Shanghai, China). Lipofectamine 2000 (Thermo Fisher Scientific, Inc.) was used to perform cell transfection, in accordance with the manufacturer's protocol. Cells were collected at $48 \mathrm{~h}$ post-transfection and grouped as follows: Control, miR-NC (100 nmol/l), miR-365b-3p mimic (100 nmol/l), PDGF-BB (20 ng/ml), PDGF-BB + miR-NC and PDGF-BB + miR-365b-3p mimic. For the PDGF-BB + miR-NC and PDGF-BB + miR-365b-3p mimic groups, the PDGF-BB was added at $24 \mathrm{~h}$ post-transfection. The sequences of mimics were as follows: miR-365b-3p mimic sense, 5'-UAA UGCCCCUAAAAAUCCUUAU-3' and antisense, 5'-AAG GAU UUU UAGGGGCAUUATT-3'; negative control mimic sense, 5'-UUCUCCGAACGUGUCACGUTT-3' and antisense, 5'-ACGUGACACGUUCGGAGAATT-3'.

Reverse transcription-quantitative polymerase chain reaction (RT-qPCR). Total RNA was extracted using TRIzol reagent (Invitrogen; Thermo Fisher Scientific, Inc.). For miR, the TaqMan MicroRNA Reverse Transcription kit (Takara Biotechnology Co., Ltd., Dalian, China) was used to obtain cDNA and a TaqMan Reverse Transcription kit (Takara Biotechnology Co., Ltd.) was used for mRNA. RT-qPCR was performed using a Perfect Real Time SYBR Premix Ex Taq kit
(Takara Biotechnology Co., Ltd.) with an ABI 7500 thermocycler (Thermo Fisher Scientific, Inc.). All kits were used according to the manufacturer's protocol. The reaction conditions for PCR were as follows: Pre-degeneration at $95^{\circ} \mathrm{C}$ for $3 \mathrm{~min}$ and 40 cycles of $95^{\circ} \mathrm{C}$ for $30 \mathrm{sec}$ and $60^{\circ} \mathrm{C}$ for $30 \mathrm{sec}$. Independent experiments were repeated three times. The relative expression levels of mRNAs were analyzed using the $2^{-\Delta \Delta C q}$ method (19). U6 and GAPDH were used as control for the expression of miR-365b-3p and ADAMTS1, respectively. The primers used were as follows: miR-365b-3p stem-loop primer, 5'-GTCGTATCCAGTGCAGGGTCCGAGGTGCAC TGGATACGACATAAGG-3', forward, 5'-TAATGCCCCTAA AAAT-3' and reverse, 5'-CCAGTGCAGGGTCCGAGGT-3'; U6 stem-loop primer, 5'-GTCGTATCCAGTGCAGGGTCCGAG GTGCACTGGATACGACAAAATATGG-3', forward, 5'-TGC GGGTGCTCGCTTCGGCAGC-3' and reverse, 5'-CCAGTG CAGGGTCCGAGGT-3'; ADAMTS1 forward, 5'-GGATGG CTGATGTTGGAA-3' and reverse, 5'-CATTAAGGCTGG CACACT-3'; and GAPDH forward, 5'-CTGGGCTACACT GAGCACC-3' and reverse, 5'-AAGTGGTCGTTGAGGGCA ATG-3'.

Western blot analysis. Total protein extraction was performed using radioimmunoprecipitation assay lysis buffer (Beyotime Institute of Biotechnology, Haimen, China). The protein concentration was measured using the BCA kit (Bio-Rad Laboratories, Inc., Hercules, CA, USA). Protein samples (20 $\mu \mathrm{g} /$ lane) were separated using $10 \%$ SDS-PAGE and blotted onto polyvinylidene difluoride membranes (Thermo Fisher Scientific, Inc.). The membranes were blocked in 5\% non-fat milk for $1 \mathrm{~h}$ at room temperature, followed by incubation overnight at $4{ }^{\circ} \mathrm{C}$ with the indicated antibodies against ADAMTS1 (cat. no. MAB 1810; 1:1,000; EMD Millipore; Billerica, MA, USA) and GAPDH (cat. no. AB 2302; 1:2,000; EMD Millipore). Subsequently, the membranes were incubated with secondary rabbit anti-mouse IgG-horseradish peroxidase antibody (cat. no. sc-358914; 1:5,000; Santa Cruz Biotechnology, Inc., Dallas, TX, USA) at room temperature for a further $2 \mathrm{~h}$. Chemiluminescent signals were visualized using the enhanced chemiluminescence detection reagent (EMD Millipore). The relative protein expression was analyzed using ImageJ software 1.4 (National Institutes of Health, Bethesda, MD, USA).

Cell Counting kit-8 (CCK-8) assay. The CCK-8 assay was used to assess cell proliferation. For the CCK- 8 assay, cells were seeded in 96-well plates $\left(5 \times 10^{3}\right.$ cells/well) and incubated at $37^{\circ} \mathrm{C}$ for $24 \mathrm{~h}$. Cells were then treated with PDGF-BB and transfected with miR-365b-3p mimic or NC. At $0,24,48$ and $72 \mathrm{~h}$ post-PDGF-BB treatment and/or transfection, cell proliferation indices were measured using a CCK- 8 kit (cat no. C0038; Beyotime Institute of Biotechnology, Shanghai, China), according to the manufacturer's protocol. The optical density was measured at $450 \mathrm{~nm}$.

Transwell and wound healing assays. Transwell and wound healing assays were used to assess cell migration. For the Transwell assay, cells of each groups (control, miR-NC, miR-365b-3p mimic, PDGF-BB, PDGF-BB + miR-NC and PDGF-BB + miR-365b-3p mimic) were seeded in the upper chambers with $200 \mu \mathrm{l}$ serum-free DMEM at density 
$\mathbf{A}$

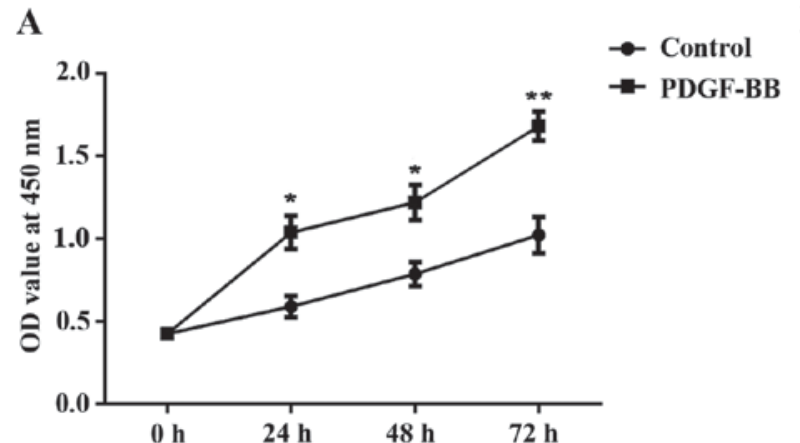

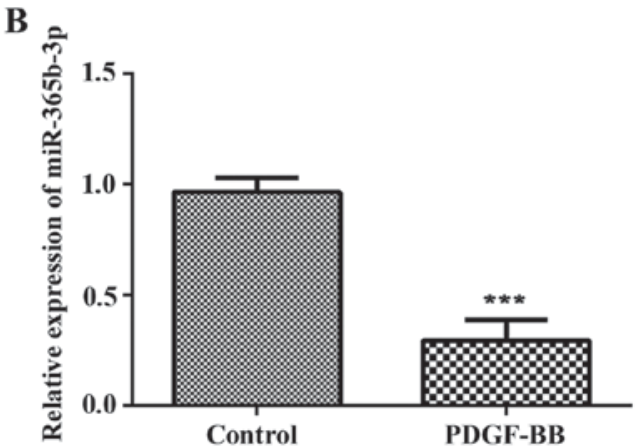

Figure 1. PDGF-BB treatment promotes HCASMC proliferation and inhibits miR-365b-3p expression. HCASMCs were treated with PDGF-BB for 6 h. (A) Cell proliferation of HCASMCs was measured using the CCK-8 assay. (B) Reverse transcription-quantitative polymerase chain reaction was conducted to evaluate miR-365b-3p expression levels. ${ }^{*} \mathrm{P}<0.05,{ }^{* *} \mathrm{P}<0.01$ and ${ }^{* * * *} \mathrm{P}<0.001$ vs. Control. PDGF-BB, platelet-derived growth factor-BB. HCASMCs, human coronary artery smooth muscle cells; miR, microRNA; OD, optical density.

of $5 \times 10^{3}$ cells $/ \mathrm{ml}$, and the lower chamber was filled with $500 \mu \mathrm{l}$ serum-free DMEM. Following an incubation time of $24 \mathrm{~h}$, the migrated cells on the lower face of the chamber membrane were fixed at room temperature for $30 \mathrm{~min}$ with $4 \%$ formaldehyde and stained with $0.1 \%$ crystal violet at room temperature for $30 \mathrm{~min}$. Cells were counted under a light microscope (magnification, x200; Nikon Corporation, Tokyo, USA).

For the wound healing assay, HCASMCs $\left(5 \times 10^{3}\right.$ cells/well $)$ were seeded into 6 -well plates. At $24 \mathrm{~h}$ post-transfection, a scratched wound was created using a pipette tip. The migrated cells were determined under an inverted microscope at 0 and 24 h. Image Pro Plus software (version 6.0; Media Cybernetics, Inc., Rockville, MD, USA) was used to measure the widths of the scratch wounds.

Dual-luciferase reporter assay. TargetScan Human 3.1 (www.targetscan.org) was used to predict the potential target of miR-365b-3p. The mutant (MUT) type of AKT2 3'-UTR was constructed using a Fast Mutagenesis System kit (TransGen Biotech, Beijing, China), according to the manufacturer's protocol. The wild type (WT) or MUT type of AKT2 3'-UTR was then inserted into the Firefly luciferase reporter vector pGL3-promoter (Promega Corporation, Madison, WI, USA) to generate the recombinant vector pGL3-ADAMTS1-3'-UTR-WT (3'-UTR-WT) or pGL3-ADAMTS1-3'-UTR-MUT (3'-UTR-MUT). HCASMCs were cultured on 24-well plates for $24 \mathrm{~h}$ prior to co-transfection with $50 \mathrm{ng}$ of 3'-UTR-WT or 3'-UTR-MUT vector and $20 \mu \mathrm{M}$ miR-365b-3p mimics or NC control. At $48 \mathrm{~h}$ post-transfection, the Dual-Luciferase Reporter Assay System (Promega Corporation) was used to determine the luciferase activity, and the Renilla luciferase activity was normalized to the Firefly luciferase activity.

Statistical analysis. Data are presented as the mean \pm standard deviation. GraphPad Prism (version 6; GraphPad software, Inc., La Jolla, CA, USA) was used to perform the statistical analyses. The differences between two groups were analyzed using the Student's t-test. Comparisons between multiple groups were determined by one-way analysis of variance followed by Bonferroni's multiple comparison tests. $\mathrm{P}<0.05$ was considered to indicate a statistically significant difference.

\section{Results}

PDGF-BB treatment promotes HCASMC proliferation and migration and inhibits miR-365b-3p expression. In the present study, HCASMCs were treated with PDGF-BB for $6 \mathrm{~h}$. Cells without any treatment were used as the control group. CCK-8 analysis revealed that the proliferation of HCASMCs was significantly increased in the PDGF-BB group compared with the control group at 24, 48 and 72 h post-PDGF-BB treatment (Fig. 1A). RT-qPCR assay was used to detect the expression of miR-365b-3p. The results indicated that the expression of miR-365b-3p was significantly decreased in the PDGF-BB group compared with that of the control (Fig. 1B). These data demonstrated that PDGF-BB treatment promotes cell proliferation and migration of HCASMCs and inhibits the expression of miR-365b-3p.

Overexpression of miR-365b-3p inhibits HCASMC proliferation and migration. To explore the function of miR-365b-3p in HCASMCs, miR-365b-3p mimic was transfected in HCASMCs with or without PDGF-BB treatment, and NC mimic was used as negative control (Fig. 2A). A CCK-8 assay revealed that overexpression of miR-365b-3p in HCASMCs with or without PDGF-BB treatment significantly inhibited cell proliferation when compared with $\mathrm{NC}$ or PDGF-BB+NC groups, respectively, at 24, 48 and $72 \mathrm{~h}$ (Fig. 2B). Transwell assays were performed to assess cell migration. The results indicated that overexpression of miR-365b-3p significantly attenuated the upregulation of PDGF-BB-induced HCASMC migration (Fig. 3). The wound healing assay exhibited the similar results (Fig. 3). Taken together, these results suggested that overexpression of miR-365b-3p inhibits HCASMC proliferation and migration.

miR-365b-3p is responsible for the PDGF-BB-mediated increase of ADAMTS1 expression. In order to further study the underlying mechanism, western blot analysis and RT-qPCR were performed to detect the expression levels of ADAMTS1. The data indicated that PDGF-BB treatment significantly increased ADAMTS1 expression at protein and mRNA levels (Fig. 4). However, the expression of ADAMTS1 protein was significantly decreased in the miR-365b-3p 

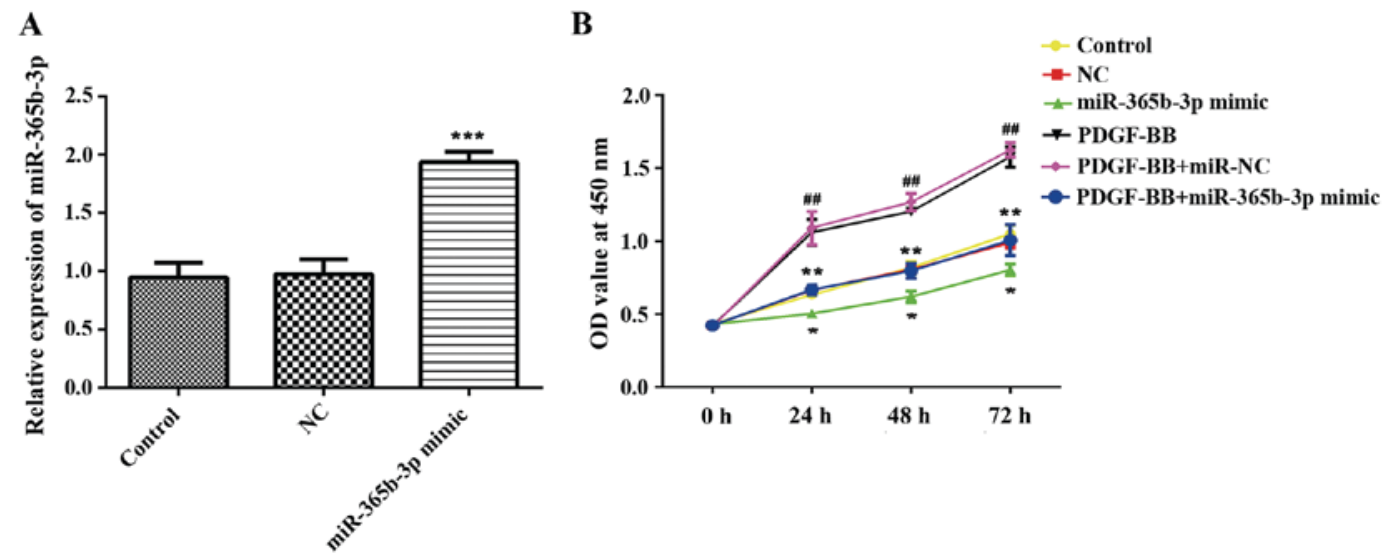

Figure 2. Overexpression of miR-365b-3p significantly attenuates PDGF-BB-induced HCASMCs proliferation. (A) miR-365b-3p mimic was transfected in HCASMCs, and reverse transcription-quantitative polymerase chain reaction was conducted to investigate the expression levels of miR-365b-3p. ${ }^{* * * *} \mathrm{P}<0.001$ vs. Control. (B) Cell proliferation of HCASMCs was measured using the CCK-8 assay. ${ }^{*} \mathrm{P}<0.05$ and ${ }^{* *} \mathrm{P}<0.001$ vs. NC or PDGF-BB+NC; ${ }^{\# \#} \mathrm{P}<0.01$ vs. Control. PDGF-BB, platelet-derived growth factor-BB; NC, negative control; miR, microRNA; OD, optical density.

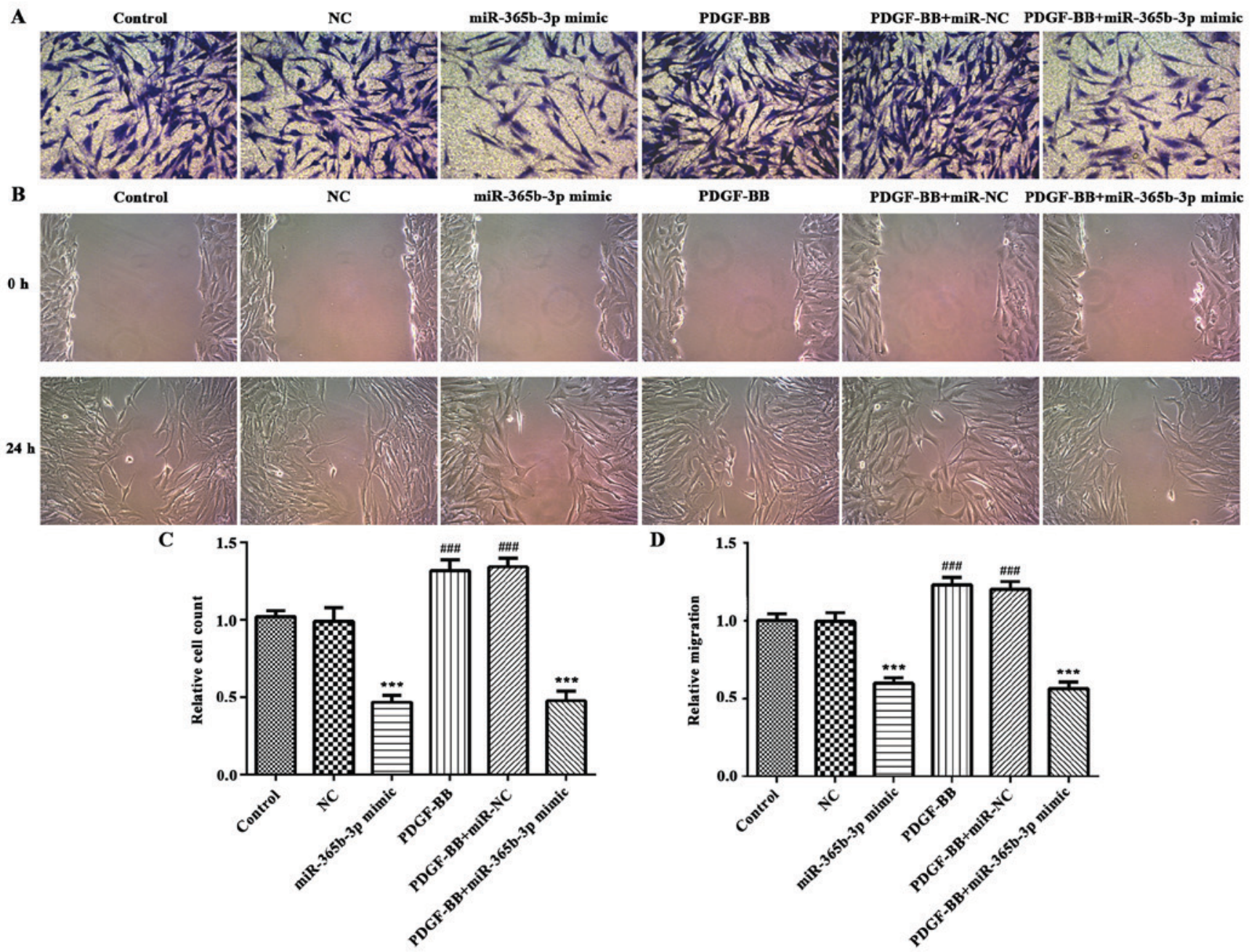

Figure 3. Overexpression of miR-365b-3p significantly attenuated PDGF-BB-induced HCASMCs migration. (A) Transwell and (B) wound healing assays were used to assess cell migration. Magnification, $\mathrm{x} 400$. The quantitative results of the (C) transwell and (D) wound healing assays are showed as bar charts. ${ }^{* * *} \mathrm{P}<0.001$ vs. NC or PDGF-BB+NC; ${ }^{\# \# \# P<0.001 ~ v s . ~ C o n t r o l . ~ P D G F-B B, ~ p l a t e l e t-d e r i v e d ~ g r o w t h ~ f a c t o r-B B ; ~ N C, ~ n e g a t i v e ~ c o n t r o l ; ~ m i R, ~ m i c r o R N A . ~}$

group compared with the NC group (Fig. 4A) and relative mRNA expression levels exhibited similar results (Fig. 4B). In the PDGF-BB+miR-365-3p group, ADAMTS1 mRNA and protein expression levels were significantly decreased compared with the PDGF-BB+NC group (Fig. 4A and B). These results revealed that miR-365b-3p may downregulate the PDGF-BB-induced expression of ADAMTS1 by contributing to the degradation of mRNA. 
A

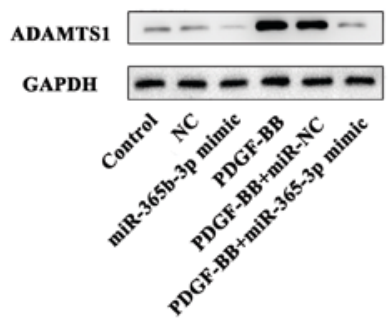

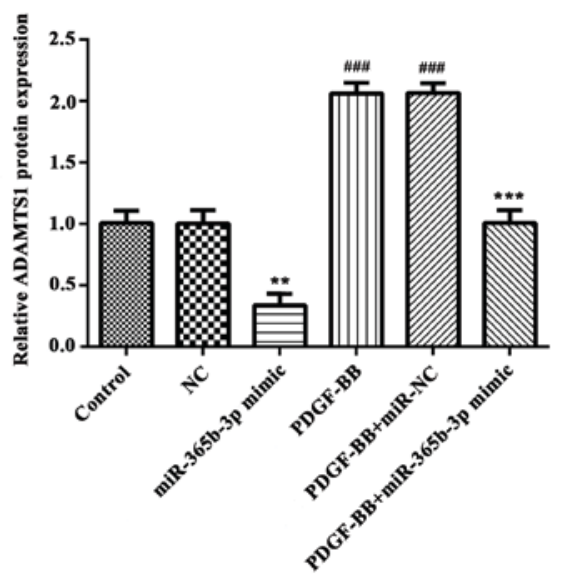

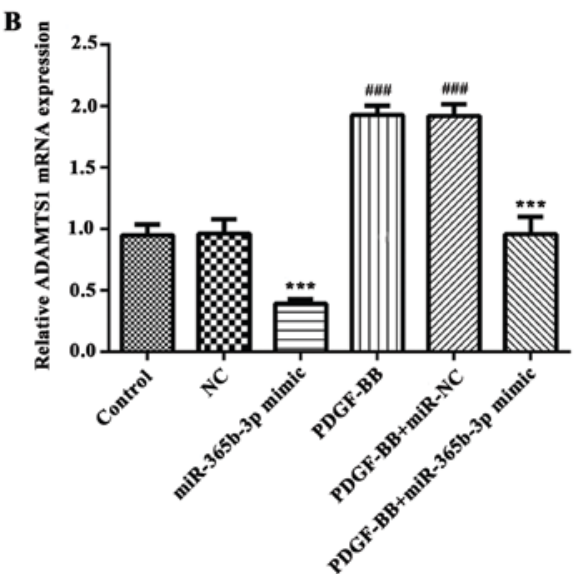

Figure 4. PDGF-BB treatment and overexpression of miR-365b-3p regulates the expression of ADAMTS1 in human coronary artery smooth muscle cells. Western blot and reverse transcription-quantitative polymerase chain reaction analysis were used to investigate the expression of ADAMTS1 (A) protein and (B) mRNA, respectively. ${ }^{* *} \mathrm{P}<0.01,{ }^{* * *} \mathrm{P}<0.001$ vs. NC or PDGF-BB+NC; ${ }^{\# \# \#} \mathrm{P}<0.001$ vs. Control. PDGF-BB, platelet-derived growth factor-BB; NC, negative control; miR, microRNA; ADAMTS1, a disintegrin and metalloproteinase with thrombospondin motifs 1 .

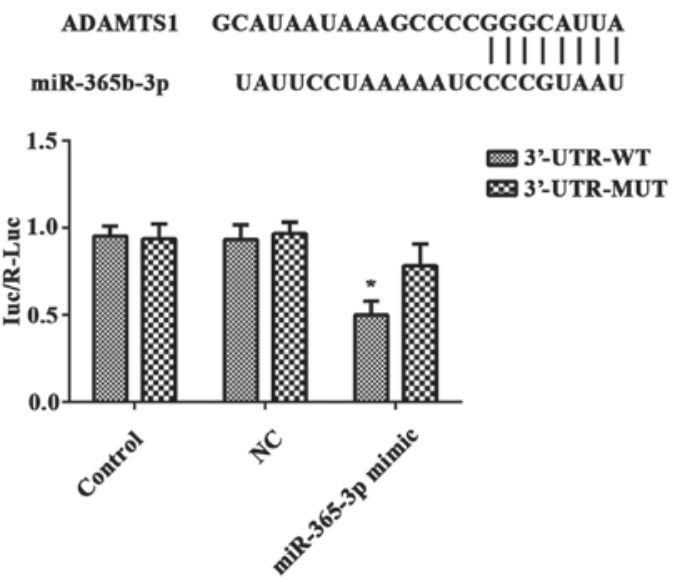

Figure 5. ADAMTS1 is a direct target gene of miR-362b-3p in HCASMCs. The putative miR-365b-3p targeted sequences in the 3'-UTR of ADAMTS1 mRNA is indicated. Dual-luciferase reporter assay was performed to analyze the relative luciferase activity in HCASMCs. Results are presented as fold change. ${ }^{*} \mathrm{P}<0.05$ vs. miR-365b-3p mimic (3'-UTR-MUT) group. 3'UTR, 3'-untranslated region; PDGF-BB, platelet-derived growth factor-BB; NC, negative control; miR, microRNA; ADAMTS1, a disintegrin and metalloproteinase with thrombospondin motifs 1 ; HCASMCs, human coronary artery smooth muscle cells.

ADAMTS1 is a direct target of miR-365b-3p. Protein and mRNA experiments indicated that ADAMTS1 may be a target gene of miR-365b-3p. To confirm this prediction, the recombinant vector pGL3-ADAMTS1-3'-UTR-WT (3'-UTR-WT) or pGL3-ADAMTS1-3'-UTR MUT type (3'-UTR-MUT) and miR-365b-3p mimic or NC mimic were co-transfected in HCASMCs, and a luciferase reporter gene assay was performed. The luciferase activity was significantly downregulated in miR-365b-3p mimic-transfected HCASMCs transfected with 3'-UTR-WT luciferase reporter plasmid when compared with those transfected with 3'-UTR-MUT luciferase reporter plasmid (Fig. 5). However, no significant difference was identified between the groups transfected with the NC mimic (Fig. 5). These findings indicated that miR-365b-3p directly binds to the 3'-UTR of ADAMTS1 mRNA in HCASMCs.

\section{Discussion}

In the present study, it was demonstrated that treatment of PDGF-BB promoted HCASMC proliferation and migration. Jönsson-Rylander et al (17) have suggested that ADAMTS1 promotes the proliferation and migration of VSMCs. Considering this, western blot analysis and RT-qPCR assays were performed to assess ADAMTS1 expression in the present study. The results suggested that PDGF-BB treatment decreased ADAMTS1 expression at protein and mRNA levels. Rescue experiments indicated that PDGF-BB treatment significantly downregulated the expression of miR-365b-3p in HCASMCs. TargetScan Human 3.1 was used to identify that ADAMTS1 is a potential target of miR-365b-3p. Based on the findings, miR-365b-3p mimic was transfected in HCASMCs to further explore the potential role of this miR in atherosclerosis. Western blot analysis and RT-qPCR assay results revealed that miR-365b-3p inhibited ADAMTS1 expression at protein and mRNA levels. The dual-luciferase reporter assay results confirmed that miR-365b-3p could directly bind to ADAMTS1. Furthermore, functional assay results indicated that miR-365b-3p inhibited HCASMC proliferation and migration. Taken together, the present findings suggested that overexpression of miR-365b-3p significantly attenuated PDGF-BB-induced proliferation and migration of HCASMCs through directly targeting ADAMTS1.

The extracellular matrix (ECM) is associated with various physiological and pathological conditions, including embryogenesis, cell-to-cell interactions, hemostasis, cell migration and apoptosis $(20,21)$. ADAMTS1 remodels the ECM through catalyzing proteoglycan degradation (22). Since destruction of ECM is thought to be responsible for the initiation of cancer $(23,24)$, multiple studies have focused on the function of ADAMTS1 in cancer. Dysregulation of ADAMTS1 have been reported in various types of cancer, including prostate, breast, lung, colon and liver cancer (25-30). Studies have revealed that ADAMTS1 has a role in the development of atherosclerosis $(17,31)$. Atherosclerosis is a complicated process stimulated by environmental and genetic factors (18) that is the leading cause of coronary heart disease, cerebral 
infarction and peripheral vascular disease (3). Subendothelial retention of atherogenic lipoproteins serves crucial roles in the initiation of atherosclerosis (32). Versican, a proteoglycan substrate of ADAMTS1, has a been demonstrated to have a critical role in this process. Notably, the degradation of versican by ADAMTS1 has been revealed to contribute to atherosclerosis (33). A recent study conducted by Vorkapic et al (34) has also demonstrated the association between the cleavage of ADAMTS1 substrates and atherosclerosis development. Furthermore, in an acute aortic dissection (AAD) mouse model, it was suggested that ADAMTS1 promotes the progression of AAD by degrading versican in macrophages and neutrophils (35). Jönsson-Rylander et al (17) suggested that ADAMTS1 may promote VSMC migration by cleaving ECM proteins in the mouse carotid artery flow cessation model. Taken together, a growing number of studies suggest that ADAMTS1 has a crucial role in the initiation and development of atherosclerosis. Although numerous animal model and tissue experimentation studies have investigated ADAMTS1, the underlying mechanisms at the molecular level are still poorly understood.

miRs are non-coding RNAs that are small in size but have significant effects. It is well acknowledged that miRs are involved in gene expression at the post-transcriptional level and serve a role in in plethora of biological processes $(10,36)$. In recent years, miRs have received much attention for their function in cardiovascular disease, including atherosclerosis $(37,38)$. With further study, a number of miRs have been identified to be associated with the regulation of ADAMTS1. For example, a previous study suggested that the expression of ADAMTS1 was inhibited by miR-142 in human endothelial progenitor cells, and the following upregulation of the endothelial nitric oxide synthase expression serves an important role in the angiogenesis (39). Previous research on breast cancer indicated that ADAMTS1 is also a target of miR-365, and downregulation of this miR inhibits cell proliferation and cell invasion (40). Other miRs, such as miR-21 (41), miR-378 (42) and miR-181d (43), have been demonstrated to have the function of regulating ADAMTS1 expression and serve their respective roles in different biological processes. However, to the best of our knowledge no research has clarified the association between ADAMTS1 and miR-365b-3p. The present findings suggested that miR-365b-3p inhibited ADAMTS1 expression by directly targeting ADAMTS1. The findings of the present study suggest the possible molecular pathway of ADAMTS1 in its involvement in atherosclerosis.

Notably, there are a few limitations of the present study. First, the experiments in the current study were investigated the mechanism at a cell level and thus, as there was not clinical data, lack further validation. Second, miRs are involved in complex regulatory networks in cells. A single mRNA molecule may be regulated by multiple miRs, and each miR may be involved in a number of gene regulatory mechanisms $(44,45)$. It is hard to say whether there are other genes regulated by the overexpression of miR-365b-3p that could impact the proliferation and migration of HCASMCs. Further studies should be performed to clarify these issues.

In conclusion the data obtained in the present study demonstrated that PDGF-BB treatment significantly downregulated the expression of miR-365b-3p in HCASMCs. Furthermore, overexpression of miR-365b-3p significantly attenuated PDGF-BB-induced HCASMC proliferation and migration through directly targeting ADAMTS1. These results provide novel evidence regarding the role of miRs in coronary atherosclerosis and advance our understanding of the mechanistic regulation of ADAMTS1.

\section{Acknowledgements}

Not applicable.

\section{Funding}

No funding was received.

\section{Availability of data and materials}

The datasets used and/or analyzed during the current study are available from the corresponding author on reasonable request.

\section{Authors' contributions}

YQ analyzed the data, wrote and revised the manuscript. YQ and NZ collected the data and designed the study.

\section{Ethics approval and consent to participate}

Not applicable.

\section{Patient consent for publication}

Not applicable.

\section{Competing interests}

The authors declare that they have no competing interests.

\section{References}

1. Weber $\mathrm{C}$ and Noels $\mathrm{H}$ : Atherosclerosis: Current pathogenesis and therapeutic options. Nat Med 17: 1410-1422, 2011.

2. Lusis AJ: Atherosclerosis. Nature 407: 233-241, 2000.

3. Roth GA, Johnson C, Abajobir A, Abd-Allah F, Abera SF, Abyu G, Ahmed M, Aksut B, Alam T, Alam K, et al: Global, regional, and national burden of cardiovascular diseases for 10 causes, 1990 to 2015. J Am Coll Cardiol 70: $1-25,2017$.

4. Johnson JL: Emerging regulators of vascular smoothmuscle cell function in the development and progression of atherosclerosis. Cardiovasc Res 103: 452-460, 2014

5. Ross R: Atherosclerosis-an inflammatory disease. N Engl J Med 340: 115-126, 1999.

6. Hutcheson JD, Goettsch C, Bertazzo S, Maldonado N, Ruiz JL, Goh W, Yabusaki K, Faits T, Bouten C, Franck G, et al: Genesis and growth of extracellular-vesicle-derived microcalcification in atherosclerotic plaques. Nat Mater 15: 335-343, 2016.

7. Millette E, Rauch BH, Kenagy RD, Daum G and Clowes AW: Platelet-derived growth factor-BB transactivates the fibroblast growth factor receptor to induce proliferation in human smooth muscle cells. Trends Cardiovasc Med 16: 25-28, 2006

8. Raines EW: PDGF and cardiovascular disease. Cytokine Growth Factor Rev 15: 237-254, 2004.

9. Bartel DP: MicroRNAs: Genomics, biogenesis, mechanism, and function. Cell 116: 281-297, 2004.

10. Moss EG: MicroRNAs: Hidden in the genome. Curr Biol 12: R138-R140, 2002. 
11. Yang X, Dong M, Wen H, Liu X, Zhang M, Ma L, Zhang C, Luan X, Lu H and Zhang Y: MiR-26a contributes to the PDGF-BB-induced phenotypic switch of vascular smooth muscle cells by suppressing Smad1. Oncotarget 8: 75844-75853, 2017.

12. Yang J, Zeng P, Yang J, Liu X, Ding J, Wang H and Chen L: MicroRNA-24 regulates vascular remodeling via inhibiting PDGF-BB pathway in diabetic rat model. Gene 659: 67-76, 2018

13. Chen $\mathrm{C}$, Yan $\mathrm{Y}$ and Liu $\mathrm{X}$ : microRNA-612 is downregulated by platelet-derived growth factor-BB treatment and has inhibitory effects on vascular smooth muscle cell proliferation and migration via directly targeting AKT2. Exp Ther Med 15: 159-165, 2018.

14. Zhang RH, Sui L, Hong XJ, Yang M and Li WM: MiR-448 promotes vascular smooth muscle cell proliferation and migration in through directly targeting MEF2C. Environ Sci Pollut Res Int 24: 22294-22300, 2017.

15. Wang J, Wang X, Wu G, Hou D and Hu Q: miR-365b-3p, down-regulated in retinoblastoma, regulates cell cycle progression and apoptosis of human retinoblastoma cells by targeting PAX6. FEBS Lett 587: 1779-1786, 2013.

16. Tang BL: ADAMTS: A novel family of extracellular matrix proteases. Int J Biochem Cell Biol 33: 33-44, 2001.

17. Jönsson-Rylander AC, Nilsson T, Fritsche-Danielson R, Hammarström A, Behrendt M, Andersson JO, Lindgren K, Andersson AK, Wallbrandt P, Rosengren B, et al: Role of ADAMTS-1 in atherosclerosis: Remodeling of carotid artery, immunohistochemistry, and proteolysis of versican. Arterioscler Thromb Vasc Biol 25: 180-185, 2005.

18. Li M, Liu Q, Lei J, Wang X, Chen X and Ding Y: miR-362-3p inhibits the proliferation and migration of vascular smooth muscle cells in atherosclerosis by targeting ADAMTS1. Biochem Biophys Res Commun 493: 270-276, 2017.

19. Livak KJ and Schmittgen TD: Analysis of relative gene expression data using real-time quantitative PCR and the 2(-Delta Delta C(T)) method. Methods 25: 402-408, 2001

20. Lu P, Weaver VM and Werb Z: The extracellular matrix: A dynamic niche in cancer progression. J Cell Biol 196: 395-406, 2012.

21. Vong S and Kalluri R: The role of stromal myofibroblast and extracellular matrix in tumor angiogenesis. Genes Cancer 2: $1139-1145,2011$.

22. Kuno K, Okada Y, Kawashima H, Nakamura H, Miyasaka M, Ohno H and Matsushima K: ADAMTS-1 cleaves a cartilage proteoglycan, aggrecan. FEBS Lett 478: 241-245, 2000.

23. Rucci N, Sanità P and Angelucci A: Roles of metalloproteases in metastatic niche. Curr Mol Med 11: 609-622, 2011.

24. Hart IR and Saini A: Biology of tumour metastasis. Lancet 339: 1453-1457, 1992.

25. Tan Ide A, Ricciardelli C and Russell DL: The metalloproteinase ADAMTS1: A comprehensive review of its role in tumorigenic and metastatic pathways. Int J Cancer 133: 2263-2276, 2013.

26. Gustavsson H, Tesan T, Jennbacken K, Kuno K, Damber JE and Welén K: ADAMTS1 alters blood vessel morphology and TSP1 levels in LNCaP and LNCaP-19 prostate tumors. Bmc Cancer 10: $288,2010$.

27. Fletcher SJ, Sacca PA, Pistone-Creydt M, Coló FA, Serra MF, Santino FE, Sasso CV, Lopez-Fontana CM, Carón RW, Calvo JC and Pistone-Creydt V: Human breast adipose tissue: Characterization of factors that change during tumor progression in human breast cancer. J Exp Clin Cancer Research 36: 26, 2017.

28. Kohno T, Otsuka A, Girard L, Sato M, Iwakawa R, Ogiwara H, Sanchez-Cespedes M, Minna JD and Yokota J: A Catalog of genes homozygously deleted in human lung cancer and the candidacy of PTPRD as a tumor suppressor gene. Genes Chromosomes Cancer 49: 342-352, 2010.
29. Mullany LE, Herrick JS, Wolff RK and Slattery ML: Single nucleotide polymorphisms within MicroRNAs, MicroRNA targets, and MicroRNA biogenesis genes and their impact on colorectal cancer survival. Genes Chromosomes Cancer 56: 285-295, 2017.

30. Lee YJ, Koch M, Karl D, Torres-Collado AX, Fernando NT, Rothrock C, Kuruppu D, Ryeom S, Iruela-Arispe ML and Yoon SS: Variable inhibition of thrombospondin 1 against liver and lung metastases through differential activation of metalloproteinase ADAMTS1. Cancer Res 70: 948-956, 2010.

31. De Schutter A, Lavie CJ and Milani RV: The impact of obesity on risk factors and prevalence and prognosis of coronary heart disease-the obesity paradox. Prog Cardiovasc Dis 56: 401-408, 2014.

32. Williams KJ: Arterial wall chondroitin sulfate proteoglycans: Diverse molecules with distinct roles in lipoprotein retention and atherogenesis. Curr Opin Lipidol 12: 477-487, 2001.

33. Wight TN: Versican: A versatile extracellular matrix proteoglycan in cell biology. Curr Opin Cell Biol 14: 617-623, 2002.

34. Vorkapic E, Folkesson M, Magnell K, Bohlooly YM, Länne T and Wågsäter D: ADAMTS-1 in abdominal aortic aneurysm. PLoS One 12: e0178729, 2017.

35. Gao Y, Wu W, Yu C, Zhong F, Li G, Kong W and Zheng J: A disintegrin and metalloproteinase with thrombospondin motif 1 (ADAMTS1) expression increases in acute aortic dissection. Sci China Life Sci 59: 59-67, 2016.

36. Wahid F, Shehzad A, Khan T and Kim YY: MicroRNAs: Synthesis, mechanism, function, and recent clinical trials. Biochim Biophys Acta 1803: 1231-1243, 2010.

37. Bayoumi AS, Aonuma T, Teoh JP, Tang YL and Kim IM: Circular noncoding RNAs as potential therapies and circulating biomarkers for cardiovascular diseases. Acta Pharmacol Sin 39: 1100-1109, 2018.

38. Pordzik J, Pisarz K, De Rosa S, Jones AD, Eyileten C, Indolfi C, Malek L and Postula M: The potential role of platelet-related microRNAs in the development of cardiovascular events in high-risk populations, including diabetic patients: A review. Front Endocrinol (Lausanne) 9: 74, 2018.

39. Zhang HW, Li H, Yan H and Liu BL: MicroRNA-142 promotes the expression of eNOS in human peripheral blood-derived endothelial progenitor cells in vitro. Eur Rev Med Pharmacol Sci 20: 4167-4175, 2016.

40. Li M, Liu L, Zang W, Wang Y, Du Y, Chen X, Li P, Li J and Zhao G: miR365 overexpression promotes cell proliferation and invasion by targeting ADAMTS-1 in breast cancer. Int J Oncol 47: 296-302, 2015.

41. Liu L, Yin H, Huang M, He J, Yi G, Wang Z and Qian H: miR-21 promotes pulmonary fibrosis in rats via down-regulating the expression of ADAMTS-1. Xi Bao Yu Fen Zi Mian Yi Xue Za Zhi 32: 1636-1640, 2016 (In Chinese)

42. Pan B, Toms D, Shen W and Li J: MicroRNA-378 regulates oocyte maturation via the suppression of aromatase in porcine cumulus cells. Am J Physiol Endocrinol Metab 308: E525-E534, 2015.

43. Chen SZ, Ning LF, Xu X, Jiang WY, Xing C, Jia WP, Chen XL, Tang QQ and Huang HY: The miR-181d-regulated metalloproteinase Adamts1 enzymatically impairs adipogenesis via ECM remodeling. Cell Death Differ 23: 1778-1791, 2016.

44. Anglicheau D, Muthukumar T and Suthanthiran M: MicroRNAs: Small RNAs with big effects. Transplantation 90: 105-112, 2010

45. Witkos TM, Koscianska E and Krzyzosiak WJ: Practical aspects of microRNA target prediction. Curr Mol Med 11: 93-109, 2011. 